\title{
Imaging Photoelectron Transmission through Self-Assembled Monolayers: The Work-Function of Alkanethiols Coated Gold
}

\author{
Despina Fragouli and Theofanis N. Kitsopoulos* \\ Institute of Electronic Structure and Laser (FORTH) and Department of Chemistry, University of Crete,
} 71110 Heraklion-Crete, Greece

Letizia Chiodo, Fabio Della Sala, and Roberto Cingolani

National Nanotechnology Laboratory of CNR-INFM, Via Arnesano, 73100 Lecce, Italy

Supratim G. Ray and Ron Naaman

Department of Chemical Physics, Weizmann Institute of Science, Rehovot, 76100, Israel

Received November 29, 2006. In Final Form: March 8, 2007

\begin{abstract}
In this paper, we present a new approach for studying the electronic properties of self-assembled monolayers and their interaction with a conductive substrate, the low-energy photoelectron imaging spectroscopy (LEPIS). LEPIS relies on imaging of photoelectrons ejected from a conductive substrate and subsequently transmitted through organic monolayers. Using this method, we measure the relative work-function of alkanethiols of different length on gold substrate, and we are able to follow the changes occurring when the surface coverage is varied. We also computed the work-function of model alkanethiols using a plane-wave density functional theory approach, in order to demonstrate the correlation between changes in the work-function with the monolayer organization and density.
\end{abstract}

\section{Introduction}

Interfaces between metals and organic molecular assemblies play an important role in all organic electronic devices, such as sensors, ${ }^{1}$ field-effect transistors, ${ }^{2}$ light-emitting diodes, ${ }^{3}$ and single-electron devices. ${ }^{4}$ Understanding the electronic properties of metal-organic interfaces is thus of fundamental importance as metallic contacts are crucial for the ultimate device performance. There is therefore an increased interest in studying how the electronic properties of interfaces ${ }^{5}$ and how the intrinsic properties of the metal, such as the work-function, can be tailored by the chemical modification at the surface. ${ }^{6}$ Extensive studies have been performed in order to investigate the adsorbatemodified electronic properties both in the case of conductor and nonconductor surfaces. ${ }^{7-12}$

Organic films and specifically self-assembled monolayers (SAMs) have been widely used for such studies, as they can form a reproducible well-defined organic-inorganic interface.

* Corresponding author. E-mail: theo@iesl.forth.gr.

(1) Janata, J.; Josowicz, M. Nat. Mater. 2003, 2, 19-24. 117.

(2) Dimitrakopoulos, C. D.; Malenfant, P. R. L. Adv. Mater. 2002, 14, 99-

(3) Burroughes, J. H.; Bradley, D. D. C.; Brown, A. R.; Marks, R. N.; Mackay, K.; Friend, R. H.; Burns, P. L.; Holmes, A. B. Nature 1990, 347, 539-541.

(4) Tour, J. M. Acc. Chem. Res. 2000, 33, 791-804.

(5) Ishii, H.; Sugiyama, K.; Ito, E.; Seki, K. Adv. Mater 1999, 11 (8), 605625 .

(6) Crispin, X.; Geskin, V.; Crispin, A.; Cornil, J.; Lazzaroni, R.; Salaneck,

W. R.; Bredas, J.-L. J. Am. Chem. Soc. 2002, 124, 8131-8141

(7) Evans, S. D.; Ulman, A. Chem. Phys. Lett. 1990, 170, 462-466.

(8) Campbell, I. H.; Kress, J. D.; Martin, R. L.; Smith, D. L.; Barashkov, N. N.; Ferraris, J. P. Appl. Phys. Lett. 1997, 71, 3528-3530.

(9) Campbell, I. H.; Rubin, S.; Zawodzinski, T. A.; Kress, J. D.; Martin, R. L.; Smith, D. L.; Barashkov, N. N.; Ferraris, J. P. Phys. Rev. B 1996, 54, 1432114324.

(10) Zehner, R. W.; Parsons, B. F.; Hsung, R. P.; Sita, L. R. Langmuir 1999, $15,1121-1127$.

(11) Howell, S.; Kuila, D.; Kasibhatla, B.; Kubiak, C. P.; Janes, D.; Reifenberger, R. Langmuir 2002, 18, 5120-5125.

(12) Ratner, M. Nature 2000, 404 (6774), 137-138.
A particular class of SAMs, that is probably the most widely and extensively studied, is constituted by the monolayers of organothiols compounds adsorbed on noble metals, primarily gold surfaces. ${ }^{13-17}$ These kinds of systems provide dense organic ordered adlayers with many tunable parameters, such as chemical composition of the terminal groups, chain length, film thickness, and organization. All of these parameters can be systematically varied in order to exploit the detailed mechanism of the interaction of the layer with the substrate and the mechanism of electronlayer interaction.

For the determination of the changes induced by thin films on the effective work-function of the substrate, contact-potential difference (Kelvin probe) measurements, and photoelectron spectroscopies have been used. ${ }^{5,7-10,18-20} \mathrm{UV}$ photoelectron spectroscopy (UPS) and X-ray photoelectron spectroscopy (XPS) are used to follow changes in effective work-function induced by the formation of hetero-junctions, charge exchange at interfaces, and charge redistribution effects ${ }^{5,18}$. Angle-resolved photoemission measurements indicate shifts in the vacuum level due to the adsorption of a close-packed alkane layer on metal or semiconductor substrates. ${ }^{21}$ Moreover, vacuum-STM, surface $\mathrm{X}$-ray, helium scattering, UPS, and Kelvin probe studies of

(13) Troughton, E. B.; Bain, C. D.; Whitesides, G. M.; Nuzzo, R. G.; Allara, D. L.; Porter, M. D. Langmuir 1988, 4, 365-385.

(14) Nuzzo, R. G.; Allara, D. L. J. Am. Chem. Soc. 1983, 105, 4481-4483.

(15) Ulman, A. An Introduction to Ultrathin Organic Films: From LangmuirBlodgett to Self-Assembly; Academic Press: New York, 1991.

(16) Nuzzo, R. G.; Dubois, L. H.; Allara, D. L. J. Am. Chem. Soc. 1990, 112 $558-569$.

(17) Alloway, D. M.; Hofmann, M.; Smith, D. L.; Gruhn, N. E.; Graham, A. L.; Colorado, R., Jr.; Wysocki, V. H.; Lee, T. R.; Lee, P. A.; Armstrong, N. R. J. Phys. Chem. B 2003, 107, 11690-11699.

(18) Seki, K.; Hayashi, N.; Oji, H.; Ito, E.; Ouchi, Y.; Ishii, H. Thin Solid Films 2001, 393, 298-303.

(19) Vilan, A.; Shanzer, A.; Cahen, D. Nature 2000, 404, 166-168.

(20) Ashkenasy, G.; Cahen, D.; Cohen, R.; Shanzer, A.; Vilan, A. Acc. Chem. Res. 2002, 35, 121-128.

(21) Seki, K.; Ueno, N.; Karlsson, U. O.; Engelhardt, R.; Koch, E.-E. Chem. Phys. 1986, 105, 247-265. 
alkanethiol monolayers on $\mathrm{Au}(111)$ have shown that substituting the terminal $-\mathrm{CH}_{3}$ group with a $-\mathrm{CF}_{3}$ group introduces a significant Columbic energy barrier to charge injection, confirming that electrical dipoles that exist both at the metal-organic interface and at the organic-vacuum interface provoke changes in the effective work-function. ${ }^{17,22}$

For the study of electron transmission through organic thin films adsorbed on metal substrates, angle-resolved photoelectron spectroscopy was used with tunable UV light sources, in which both the energy and the momentum of electrons photoejected from the metal are resolved, after the electrons passed through the organic layer. ${ }^{23-25}$ In this method, the low-energy photoelectron transmission spectroscopy (LEPS), ${ }^{26}$ the transmission yield can be measured as a function of the electron energy, the film structure, etc. The above-mentioned studies showed that electrons with energy higher than $0.8 \mathrm{eV}$ pass very efficiently through organized organic films ${ }^{23,24}$ and revealed that the photoelectron transmission probability depends on the periodicity of the organization of the layers, both parallel and perpendicular to the velocity of the transmitted electrons. ${ }^{25}$

In a complementary method, the low-energy electrontransmission (LEET) spectroscopy, mono-energetic electrons are injected into the layer from the vacuum side and are collected on the conductive substrate. ${ }^{27}$ A closely related experimental technique is the high-resolution electron energy loss spectroscopy (HREELS), ${ }^{28}$ where a monochromatic electron beam hits an adsorbed molecular layer from the vacuum side.

In the present study we introduce the low-energy photoelectron imaging spectroscopy (LEPIS) as a new way to probe the electronic properties of SAMs, close to the Fermi level. This method relies on imaging of photoelectrons produced by the metal substrates after UV irradiation. Following the ejection of the electrons from the substrate, they are transmitted through the organic monolayer. We show that this method allows relative work-function measurements of the monolayer coated substrate as well as the study of the kinetics of the formation of the monolayer of the alkanethiol from solution.

Results that are presented here relate the kinetics of the monolayer formation with the relative changes in the workfunction. Studies on SAM formation kinetics have been performed in the past using ellipsometry, contact angle measurements, ${ }^{29,30}$ infrared and fluorescence spectroscopy, ${ }^{31}$ surface acoustic wave devices, ${ }^{32}$ second-harmonic generation, ${ }^{33}$ near-edge X-ray absorption fine structure, ${ }^{34}$ quartz crystal microbalances, ${ }^{35}$ and surface stress measurements. ${ }^{36}$ These studies revealed that the growth rate is proportional to the number of unoccupied adsorption sites, in agreement with the simple first-order Langmuir adsorption

(22) Pflaum, J.; Bracco, G.; Schreiber, F.; Colorado, R. Jr.; Shmakova, O. E.; Lee, T. R.; Scoles, G.; Kahn, A. Surf. Sci. 2002, 498, 89-104.

(23) Kadyshevitch, A.; Naaman, R. Thin Solid Films 1996, 288, 139-146.

(24) Kadyshevitch, A.; Naaman, R. Phys. Rev. Lett. 1995, 74, 3443-3446.

(25) Kadyshevitch, A.; Ananthavel, S. P.; Naaman, R. J. Chem. Phys. 1997, 107, 1288-1290.

(26) Naaman, R.; Vager, Z. Acc. Chem. Res. 2003, 36, 291-299.

(27) Sanche, L. Scanning Microsc. 1995, 9, 619-656.

(28) Dannetun, P.; Schott, M.; Vilar M. Thin Solid Films 1996, 286, 321-329.

(29) Bain, C. D.; Whitesides, G. M. J. Am. Chem. Soc. 1989, 111, 7164-7175.

(30) Bain, C. D.; Troughton, E. B.; Tao, Y.-T.; Evall, J.; Whitesides, G. M.; Nuzzo, R. G. J. Am. Chem. Soc. 1989, 111, 321-335.

(31) Chen, S. C.; Frank, C. W. Langmuir 1989, 5, 978-987.

(32) Thomas, C. R.; Sun, L.; Crooks, R. M.; Ricco, A. J. Langmuir 1991, 7, $620-622$.

(33) Buck, M.; Grunze, M.; Eisert, F.; Fischer, J.; Trager, F. J. Vac. Sci. Technol. A 1992, 10 (4), 926-929

(34) Haehner, G.; Woll, C.; Buck, M.; Grunze, M. Langmuir 1993, 9, 19551958.

(35) Karpovich, D. S.; Blanchard, G. J. Langmuir 1994, 10, 3315-3322.

(36) Berger, R.; Delamarche, E.; Lang, H. P.; Gerber, C.; Gimzewski, J. K.; Meyer, E.; Güntherodt, H.-J. Science 1997, 276, 2021-2024. kinetics. ${ }^{31,33,35,37}$ Our results however show nonlinear relationship between the work-function kinetics and the coverage kinetics studied with the above-mentioned methods.

These experimental findings are supported by first-principles density-functional theory (DFT) calculations. From a theoretical point of view, the interface between SAMs and gold surfaces, in particular the $\mathrm{Au}(111)$, is one of the most investigated systems. ${ }^{38-41}$ A lot of information is available about the conformation of the adsorbate and the energetic properties of $\mathrm{CH}_{3} \mathrm{~S}$ and $\left(\mathrm{CH}_{3} \mathrm{~S}\right)_{2}$ on $\mathrm{Au}(111) .{ }^{39}$ Due to the large computational costs required for these systems, usually only short-chain thiol molecules have been investigated ab initio. In particular, being the S-metal interaction dominant in the molecular adsorption geometry, the attention has focused on $\mathrm{CH}_{3} \mathrm{~S}$ on $\mathrm{Au}(111)$, treated as a prototype for SAMs - metal interface. It is known that shortchain alkanethiols adsorbed on gold induce a work-function reduction. ${ }^{42}$ Reported values for $\mathrm{Au}$ work-function range from $4.9^{43}$ to $5.3 \mathrm{eV},{ }^{42,44}$ and the thiol induced reduction is of $0.8^{43}$ to $1.2^{42} \mathrm{eV}$, with maximum values of $1.4 \mathrm{eV}^{17}$. Here, we calculate the effect on the gold work-function of adsorbed methanthiol and of $\mathrm{CH}_{3}\left(\mathrm{CH}_{2}\right)_{5} \mathrm{SH}$ (hexanethiol), which introduces the effect of the long alkyl chain on the work-function.

\section{Experimental Details}

Materials. Crystalline gold films, $\mathrm{Au}(111)$, of thickness of 200 $\mathrm{nm}$ that are evaporated onto chromium layers of $40 \mathrm{~nm}$ on slides of borosilicate glass $(38 \times 12 \mathrm{~mm})$ were purchased from Arrandee Co. Octadecanethiol $\left(\mathrm{CH}_{3}\left(\mathrm{CH}_{2}\right)_{17} \mathrm{SH}\right)$ and dodecanethiol $\left(\mathrm{CH}_{3}\left(\mathrm{CH}_{2}\right)_{11^{-}}\right.$ $\mathrm{SH}$ ) (denoted as $\mathrm{C} 18$ and $\mathrm{C} 12$ ) were purchased from Aldrich and used without further purification, and ethanol was HPLC grade (Aldrich).

Samples Preparation. Gold surfaces were exposed for $20 \mathrm{~min}$ to radiation from a homemade ozonator, which contains a UV lamp (Jeligth Co) and generates light emission in the range of 185-579 $\mathrm{nm}$. The ozone is produced upon absorption of the $185-\mathrm{nm}$ emission by atmospheric oxygen while the organic moieties are excited and dissociated by the 254-nm radiation. The excited organic molecules react with ozone to form volatile products such as water and carbon dioxide. ${ }^{45}$ Immediately following the oxidation step, the gold-coated samples were immersed in pure ethanol for $20 \mathrm{~min} . \mathrm{CH}_{3}\left(\mathrm{CH}_{2}\right)_{17} \mathrm{SH}$ monolayers were prepared by immersion of the gold samples in a $\sim 7 \mu \mathrm{M} \mathrm{C18}$ solution in ethanol, at room temperature, for time intervals ranging from a few seconds to several hours, while $\mathrm{CH}_{3}\left(\mathrm{CH}_{2}\right)_{11} \mathrm{SH}$ monolayers were prepared by immersion of the gold samples in a $\sim 10^{-1} \mathrm{mM} \mathrm{C12}$ solution in ethanol. The samples were then rinsed thoroughly with ethanol and blow dried with a stream of nitrogen gas.

Contact-Angle (CA) Measurements. In order to confirm the presence of the monolayer on the gold substrate, the resulting films were characterized by measuring the static $\mathrm{CA}$ of nanopurified water drops of $10 \mu \mathrm{L}$ and $3 \mu \mathrm{L}$ onto the films, using a homemade CA tensiometer instrument.

Photoelectron Spectrometer. The experimental apparatus used in these studies is a home-built photoelectron imaging spectrometer

(37) Thomas, R. C.; Sun, L.; Crooks, R. M.; Ricco, A. J. Langmuir 1991, 7, $620-622$.

(38) Vargas, M. C.; Giannozzi, P.; Selloni, A.; Scoles, G. J. Phys. Chem B 2001, 105, 9509-9513.

(39) Yourdshahyan, Y.; Rappe, A. M. J. Chem. Phys. 2002, 117, 825-833. (40) Zhang, L.; Goddard, W. A.; Jiang, S. J. Chem. Phys. 2002, 117, 73427349 .

(41) Gronbeck, H.; Curioni, A.; Andreoni, W. J. Am. Chem. Soc. 2000, 122, $3839-3842$.

(42) De Renzi, V.; Rousseau, R.; Marchetto, D.; Biagi, R.; Scandolo, S.; del Pennino, U. Phys. Rev. Lett. 2005, 95, 046804.

(43) de Boer, B.; Hadipour, A.; Mandoc, M.; van Woudenbergh, T.; Blom, P. W. M. Adv. Mater. 2005, 17, 621-625.

(44) Heimel, G.; Romaner, L.; Brédas, J.-L.; Zojer, E. Phys. Rev. Lett. 2006, 96, 196806.

(45) Ron, H.; Matlis, S.; Rubinstein, I. Langmuir 1998, 14, 1116-1121. 


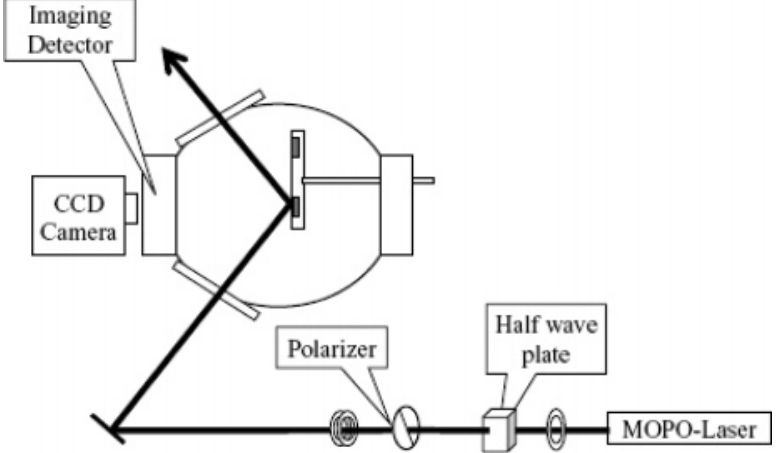

Figure 1. Schematic of the photoelectron imaging spectrometer.
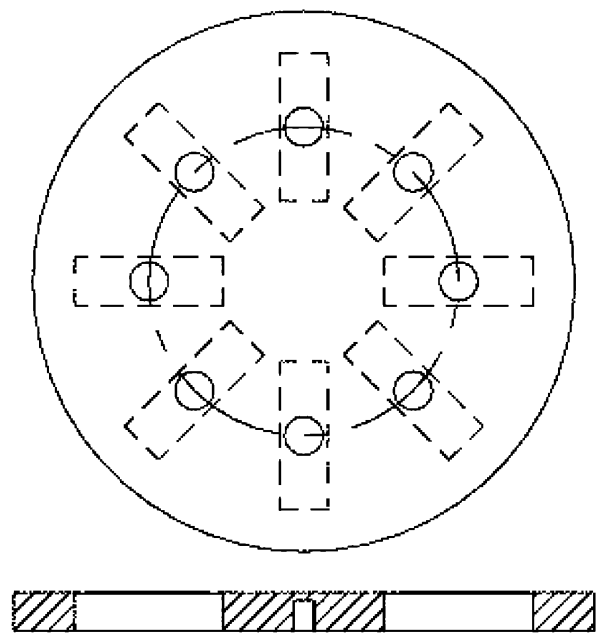

Figure 2. Detail schematic of the sample holder. It supports up to eight samples, in a fly wheel fashion. A groove that matches the dimension of the sample $(38 \times 12 \mathrm{~mm})$ is cut out of the sample holder, leaving exposed only a thin piece of metal $(0.5 \mathrm{~mm})$. The $10 \mathrm{~mm}$ hole is bored out in the center of the sample groove and it is only this region of the sample that is exposed to the laser light. The holder can be rotated without breaking vacuum. The lower part is the profile of the holder.

shown schematically in Figure 1. It consists of a vacuum chamber pumped using a $300 \mathrm{~L} / \mathrm{s}$ turbomolecular pump, and typical operating vacuum pressure of $2 \times 10^{-7} \mathrm{mbar}$. The sample holder is capable of supporting up to eight samples, in a fly wheel fashion. A groove that matches the dimension of the sample $(38 \times 12 \mathrm{~mm})$ is cut out of the sample holder, leaving exposed only a thin piece of metal (Figure 2). A $10 \mathrm{~mm}$ hole is bored out in the center of the sample groove, and it is only this region of the sample that is exposed to the laser light. Samples are placed in the groove face (SAM side) down. The holder can be rotated without breaking vacuum, thus allowing facile relative measurements of up to eight samples. The holder is made out of aluminum and is electrically isolated (floating) thus also serving as a repeller electrode. A uniform electric field is generated by placing a square grid (333 lines per in., $8 \mathrm{~cm}$ width) about $4 \mathrm{~cm}$ from the repeller. This field is used to accelerate photoelectrons generated toward a position sensitive detector consisting of a pair of microchannel plates (DelMar) coupled to a phosphor screen (Proxitronic). Photoelectron images appearing on the detector's phosphor screen are recorded using a $512 \times 512$ slow scan CCD camera (Spectra Source). The samples are irradiated using the output of MOPO (730-D10 Spectra Physics) operating at $10 \mathrm{~Hz}$ and laser powers of $\leq 0.1 \mu \mathrm{J} /$ pulse. The MOPO laser is linearly polarized, and its polarization direction can be varied using $\lambda / 2$ waveplates. The photoelectron images are typically averaged over 100 laser shots and recorded for each of the samples ${ }^{46}$ at various

(46) Dimitrov, D.; Trakhtenberg, S.; Naaman, R.; Smith, D. J.; Samartzis, P. C.; Rakitzis, T. P.; Kitsopoulos, T. N. Chem. Phys. Lett. 2000, 322, 587-591. wavelengths. Given the energy of the photon used (less than $5 \mathrm{eV}$ ), all the photoelectrons are generated from the gold substrate.

\section{Computational Details}

$\mathrm{Ab}$ initio calculations have been performed in the framework of DFT, using the plane-waves code PWSCF. ${ }^{47}$ UltraSoft Pseudopotentials ${ }^{48}$ have been chosen with wave function and charge density cutoff of 40 and 350 Ryd, respectively. The PW91 parametrization $^{49}$ for the exchange-correlation potential has been used.

The clean gold surface $\mathrm{Au}(111)$ is described by a repeated supercell, constituting of a slab of seven Au atomic layers and a vacuum region $19.23 \AA$ thick. For the work-function calculation, convergence has been carefully tested on number of atomic (up to 9) and vacuum (until 12) layers. The k-point mesh convergence has been tested until $10 \times 10 \times 1$ for clean gold and $8 \times 8 \times$ $1 \mathrm{k}$-points for the covered surface.

The coverage $\theta=1$ monolayer ( $\mathrm{mL}$ ) corresponds to one thiol molecule for three Au surface atoms, in the hexagonal $(\sqrt{3} \times$ $\sqrt{3})$ R $30^{\circ}$ adsorption geometry (see Figure 6 , panel I). The favored adsorption site for short-chain alkanethiols has been characterized to be the bridge one ${ }^{38,39}$ slightly shifted toward the fcc position. Further calculation have been performed for $\theta$ $=0.5 \mathrm{~mL}$ ( 1 molecule per $6 \mathrm{Au}$ surface atoms $)$ and $\theta=0.25$ $\mathrm{mL}$ (1 molecule per $12 \mathrm{Au}$ surface atoms) for $\mathrm{CH}_{3} \mathrm{~S}$.

The work-function is one of the more complex properties to be calculated $a b$ initio because it is quite sensitive to a lot of computational parameters convergence and to the surface structural and electronic details. The work-function $\Phi$ is calculated as the difference between the electrostatic potential in the vacuum region $V_{\mathrm{ES}}$ and the Fermi energy $E_{\mathrm{Fermi}}$ (see Figure 7). The dipole correction is included to avoid the effect of the periodicity on the electrostatic potential ${ }^{50,51}$ in the vacuum.

\section{Experimental Results}

A typical photoelectron image is shown in Figure 3a. The bright intensity spot corresponds to large photoelectron currents. The images have the greatest intensity at the center and it tails off at larger radii. The imaging plane is parallel to the surface of the target. The appearance of the image supports the conclusion that the majority of the photoelectrons are ejected in the vicinity of the normal to the surface-symmetry axis. Although ideally one would like to obtain energy resolved images of the photoelectrons perpendicular to the surface normal (i.e., the system symmetry axis), for technical reasons this is not yet possible. Hence the images obtained are non-energy resolved, but as the laser excitation energy is close to the work-function of the gold substrate, it is assumed that the photoelectron energy distribution is relatively narrow.

Intensity profiles of the images are fitted using Gaussian distribution functions as shown in Figure $3 \mathrm{~b}$. This procedure yields information on two parameters. The width of the distribution represents the width of the image and the area under the curve represents the total amount of the photoelectrons extracted from the gold surface and transmitted through the film.

By monitoring the angular distribution of the transmitted photoelectrons it has been found that the transmission is by far more efficient along the direction of the organic chains than in any other direction. ${ }^{26} \mathrm{~A}$ channelling effect is observed for electrons

(47) Baroni, S.; Corso, A. D.; de Gironcoli S.; Giannozzi P. http:// www.pwscf.org/.

(48) Vanderbilt, D. Phys. Rev. B 1990, 41, 7892-7895.

(49) Perdew, J. P. Physica B 1991, 172, 1-6

(50) Bengtsson, L. Phys. Rev. B 1999, 59, 12301-12304.

(51) Tóbik, J.; Dal Corso, A. J. Chem. Phys. 2004, 120, 9934-9941. 


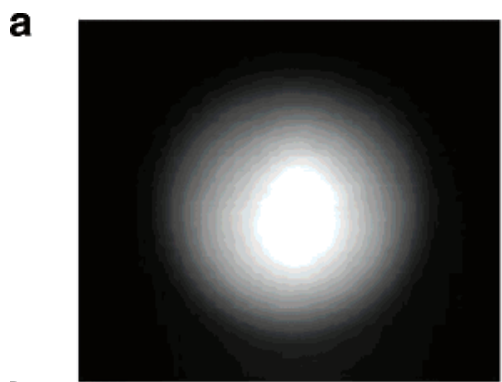

$\mathbf{b}_{2,0}$

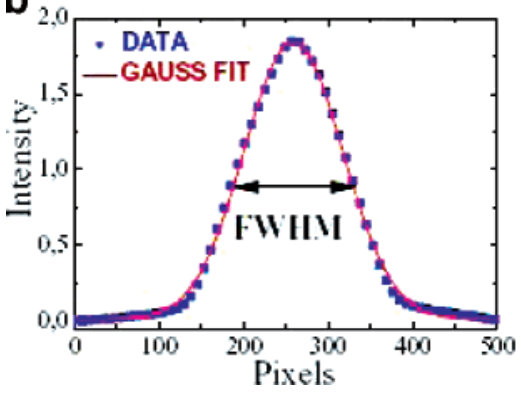

Figure 3. (a) Typical photoelectron image: the bright intensity corresponds to large photoelectron currents. The images have the greatest intensity at the center and tail of at larger radii. The imaging plane is perpendicular to the surface normal. (b) Intensity profile of the photoelectron image, taken as the average of the vertical pixel columns. The Gaussian fit of the photoelectrons intensity distributions gives the fwhm that represents the width of the image.

ejected into the organic films. These results explain the extremely high sensitivity of the transmission efficiency through organized organic films on their structural order. ${ }^{46}$

Photoelectron imaging is well-established in gas-phase experiments. ${ }^{52}$ The size (width) of the image is proportional to the speed of the photoelectrons and consequently to the root square of the kinetic energy. From conservation of energy one obtains

$$
\mathrm{eKE}=E_{\text {photon }}-\Phi
$$

where eKE is the photoelectron kinetic energy and $\Phi$ is the work-function of the sample. From the above equation it is obvious that when $\Phi$ becomes identical to the energy of the photon, eKE $=0$. To obtain the work-function of a particular sample, we plot the width of the image intensity as a function of photon energy and extrapolate this curve to zero width (i.e., zero kinetic energy). Typical results are shown in Figure 4.

We have used the photoelectron image analysis procedure to examine the trends in the change of the $\Phi$ by SAMs as a function of sample immersion time in the precursor solution. Our samples were also characterized by monitoring the surface wettability. It is well-known that the change in surface wettability reflects the quality of the monolayer. ${ }^{15,53,54}$ Figure 5 presents the dependence of the contact angle on the immersion time, for the $\mathrm{C} 18$ and $\mathrm{C} 12$ SAMs. For $\mathrm{C} 18$ the lowest value is about $84^{\circ}$, for the low coverage sample, while the maximum value is about $102^{\circ}$ for the sample with the maximum coverage. In the case of $\mathrm{C} 12$ we noticed that the highest values for the contact angles did not exceed $90^{\circ}$. This typically means that the "quality" of the monolayer is not as good as that of $\mathrm{C} 18$ and this result is expected, consistent with general observations that longer chain molecules self-assemble much more readily. ${ }^{30}$

(52) Ashfold, M. N. R.; Nahler, N. H.;Orr-Ewing, A. J.; Vieuxmaire, O. P J.; Toomes, R. L.; Kitsopoulos, T. N.;Garcia, I. A.; Chestakov, D. A.; Wu, S. M.; Parker, D. H. Phys. Chem. Chem. Phys. 2006, 8, 26.

(53) Nuzzo, R. G.; Fusco, F. A.; Allara, D. L. J. Am. Chem. Soc. 1987, 109 $2358-2368$.

(54) Allara, D. L.; Nuzzo, R. G. Langmuir 1985, 1, 45; 1985, 1, 52-66.

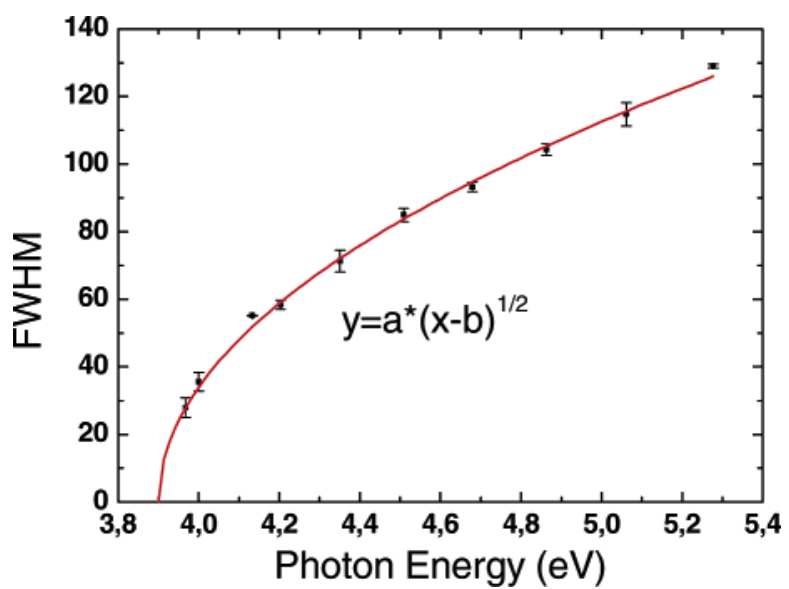

Figure 4. Plot of the fwhm of the photoelectron images vs the irradiation photon energy for a sample of bare Au. To obtain the work-function of a particular sample, the fwhm of the image intensity is plotted as a function of photon energy and extrapolate this curve to zero width. The fitting function is $y=a(x-b)^{1 / 2}$, and the fitting coefficient $b$ equals to the $\Phi$ of each sample.

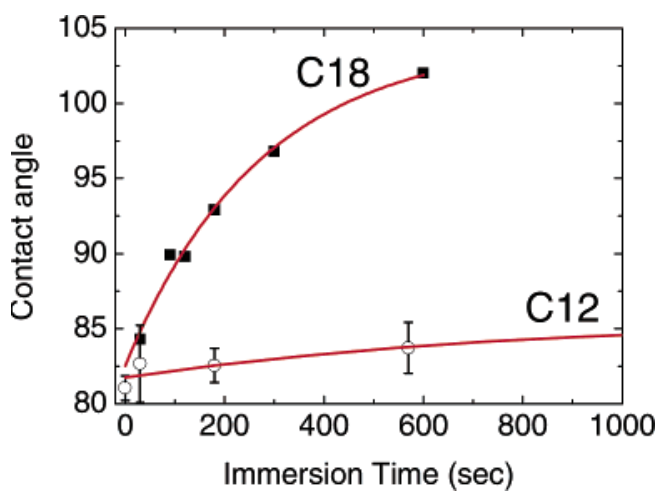

Figure 5. Plot of the water contact angle vs sample immersion times of the $\mathrm{C} 18$ and $\mathrm{C} 12$ samples, fitted by the functional form of Langmuir kinetics.

\section{Theoretical Results}

We performed calculations for $\mathrm{CH}_{3} \mathrm{~S}$ adsorption both in the bridge (Figure 6c) and the hollow site (Figure 6d). The former is the most stable configuration and actually relaxes toward the hollow site, with a small shift of $0.2 \AA$ and a tilt angle of $58^{\circ}$, while the latter is less stable by $0.29 \mathrm{eV}$.

For the larger $\mathrm{CH}_{3}\left(\mathrm{CH}_{2}\right)_{5} \mathrm{~S}$ - (henceforth denoted as $\mathrm{C} 6$ ), we assume the stable adsorption site to be the same as the methanethiol, the slightly relaxed bridge site. The C6 is placed in the bridge site, and the whole system is allowed to relax until force components are less than $0.025 \mathrm{eV} / \AA$. We obtain a shift of $0.2 \AA$ from the pure bridge site toward the fcc site, in good agreement with the results found for methanethiol (Figure 6a). The molecular axes is tilted by $32^{\circ}$ with respect to the surface normal, in quite good agreement with experimental data. ${ }^{22,30,55}$ The staggered orientation for $\mathrm{H}$ atoms has been assumed. ${ }^{39} \mathrm{~A}$ different rotation of the $\mathrm{C}$ atoms along the molecular axis (Figure 6b) has also been calculated, resulting to be less stable than configuration (a) by $0.36 \mathrm{eV}$.

The calculated work-function for the clean $\mathrm{Au}(111)$ surface is $5.11 \mathrm{eV}$, obtained carefully checking convergence over atomic and vacuum layers and k-points sampling, and in good agreement with previous experimental and theoretical results. ${ }^{42,44}$

(55) Porter, M. D.; Bright, T. B.; Allara, D. L.; Chidseyi, C. E. D. J. Am. Chem Soc. 1987, 109, 3559-3568. 

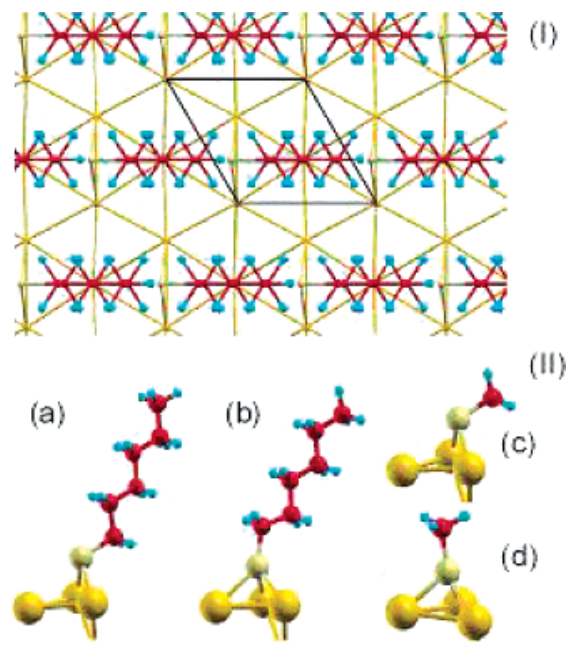

Figure 6. (I) $1 \mathrm{~mL}$ of $\mathrm{C} 6$ in the hexagonal $(\sqrt{3} \times \sqrt{3}) \mathrm{R} 30^{\circ}$ adsorption geometry on $\mathrm{Au}(111)$, the surface cell is indicated. Yellow denotes $\mathrm{Au}$ atoms, light yellow S, red C, light blue $\mathrm{H}$. (II) adsorption configurations of $\mathrm{C} 6$ and $\mathrm{CH}_{3} \mathrm{~S}$ on $\mathrm{Au}(111)$ : (a) stable bridge adsorption site for C6, (b) C6 in the bridge site with the molecular axis rotated with respect to configuration a, (c) stable bridge site for $\mathrm{CH}_{3} \mathrm{~S}$, (d) hollow site for $\mathrm{CH}_{3} \mathrm{~S}$.

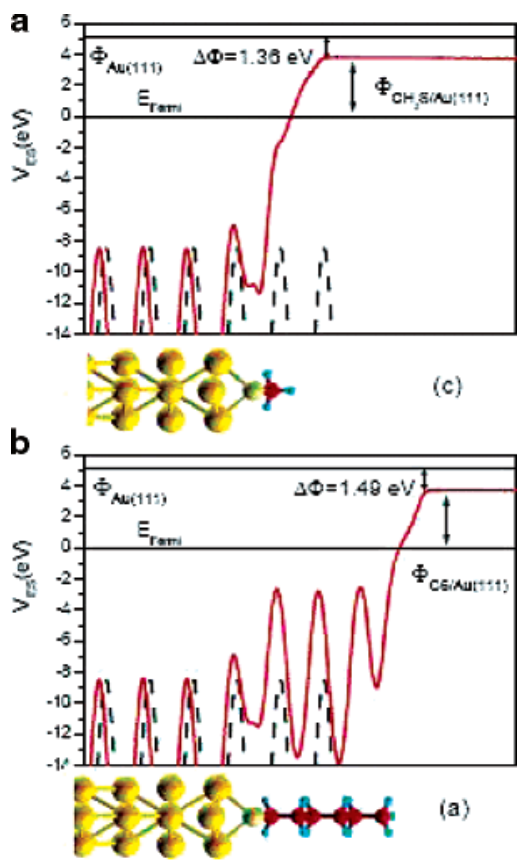

Figure 7. Behavior of plane-averaged $V_{\mathrm{ES}}$ for $\mathrm{CH}_{3} \mathrm{~S}$ (a) at $1 \mathrm{~mL}$ coverage in the bridge geometry (see Figure 6c) and for C6 (b) at $1 \mathrm{~mL}$ coverage in the geometry of Figure $6 \mathrm{a}$. The dotted black curve denote the potential of Au bulk, the solid red one denotes the electrostatic potential of the slab with the adsorbate. The Au Fermi level, the Au work-function, and the work-function changes $\Delta \Phi$ for the two hybrid systems are reported.

For the methanethiol (c), at $1 \mathrm{~mL}$ coverage, the induced workfunction reduction is $1.36 \mathrm{eV}$, reaching the value of $3.75 \mathrm{eV}$. For the long-chain molecule C6 (a), the reduction value is quite similar, $1.49 \mathrm{eV}$. In Figure 7 the electrostatic potential and the work-function changes are shown for methanethiol and C6. For the less stable adsorption configurations, for both the considered molecules, the work-function drop is larger, $2.31 \mathrm{eV}$ for the $\mathrm{C} 6$ in the (b) configuration and $2.17 \mathrm{eV}$ for $\mathrm{CH}_{3} \mathrm{~S}$ in the hollow site (d).

The values for $\theta=0.25 \mathrm{~mL}$ and $\theta=0.5 \mathrm{~mL}$ of $\mathrm{CH}_{3} \mathrm{~S}$ on $\mathrm{Au}$ (111) are 4.56 and $4.22 \mathrm{eV}$, with a $\Delta \Phi$ of 0.55 and $0.89 \mathrm{eV}$, respectively. The variation of $\Phi$ with coverage is therefore nonlinear, as it can be well reproduced by $\Phi(\theta)=\Phi_{\mathrm{SAM}}+\left(\Phi_{\mathrm{Au}}\right.$ $\left.-\Phi_{\mathrm{SAM}}\right)(1-\theta)^{n}$, with $n=1.6$.

\section{Discussion}

It has been shown that the growth kinetics of SAMs is proportional to the number of unoccupied adsorption sites, in agreement with simple first-order Langmuir adsorption kinetics: ${ }^{31,33,35,37}$

$$
\frac{\mathrm{d} \theta}{\mathrm{d} t}=-k(1-\theta)
$$

where $\theta$ is the fraction of occupied sites (or surface coverage), $t$ is the time, and $k$ is the adsorption rate constant that result from the flux of thiol molecules to the surface and the sticking coefficient. The integration of eq 2 yields the time course of the monolayer formation:

$$
\theta(t)=\theta_{\mathrm{f}}\left[1-\exp \left(-k_{\mathrm{L}} t\right)\right]
$$

where $\theta_{\mathrm{f}}$ is the coverage value for long adsorption time (i.e., the maximum coverage) while for $t=0, \theta=0$. It is well-known that surface wettability can be used for obtaining the surface coverage. ${ }^{56}$ The kinetics of the monolayer formation depends of course on the concentration of the ethanolic solution; however, it is also strongly affected by the chain length. ${ }^{15,30}$ In Figure 5 we fit our data using eq 3 and we obtain Langmuir rate constants $k_{\mathrm{L}}=4 \pm 2 \times 10^{-3} \mathrm{~s}^{-1}$ and $k_{\mathrm{L}}=1.3 \pm 1 \times 10^{-3} \mathrm{~s}^{-1}$ for the C18 and C12 SAMs, respectively. As discussed above it is clear that the C12 SAMs never reach contact angles larger than $90^{\circ}$ even for immersion time of $20000 \mathrm{~s}$. This means that the quality of the organization is pour. In other words, we believe that although the adsorbates have achieved full coverage of the gold substrate, the organization of the SAM is much worse for $\mathrm{C} 12$ as compared to the longer chain $\mathrm{C} 18$.

A very striking observation that we must address is the fact that we measure a value for the work-function of gold close to $4 \mathrm{eV}$, while the calculated theoretical value for the clean surface is $5.11 \mathrm{eV} .{ }^{42,44} \mathrm{It}$ is well-established that the work-function of $\mathrm{Au}$ surfaces is very sensitive to contaminants and XPS measurements on freshly evaporated Au surfaces exposed to laboratory atmosphere for several minutes have confirmed the presence of a carbon- and oxygen-containing contaminant layer of approximately 6 - $\AA$ thick ${ }^{30}$. Exposure to air invariably reduces the work-function ${ }^{57}$ primarily because of hydrocarbon adsorption ${ }^{58,59}$ to values as low as $4.3 \mathrm{eV} .{ }^{60,61}$ Hence we attribute our observed work-function to the relatively low vacuum $\left(10^{-7} \mathrm{mbar}\right)$ in our experimental chamber, which results in the formation of "contamination layers" by residual gases. A large percentage of these residual gases contain hydrocarbon molecules from pump oil thus explaining $\sim 1 \mathrm{eV}$ offset from the established workfunction value for $\mathrm{Au}(111)$. It is important to realize that upon adsorption of the self-assembled monolayer, the contaminants are removed by the adsorbed molecules.

To correct for "contamination layer" effects, we assume that the maximum effect will occur for a bare gold substrate since

(56) Israelachvili, J. N.; Michelle, L. G. Langmuir 1989, 5, 288.

(57) Tonck, A.; Houze, F.; Boyert, L.; Loubet, J-L; Georges, J-M. J. Phys. Condens. Matter 1991, 3, 5195-5201.

(58) Thiel, P. A.; Madey, T. E. Surf. Sci. Rep. 1987, 7, 211-385.

(59) Callen, B. W.; Griffiths, K.; Memmert, U.; Harrington, D. A.; Bushby, S. J.; Norton, P. R. Surf. Sci. 1990, 230, 159-174.

(60) Ashcroft, N. W; Mermin, N. D. Solid State Physics; Holt, Rinehart and Wilson: New York, 1976).

(61) Mamin, H. J; Guethner, P. H.; Rugar, D. Phys. Rev. Lett. 1990, 65, 19, 2418-2422. 


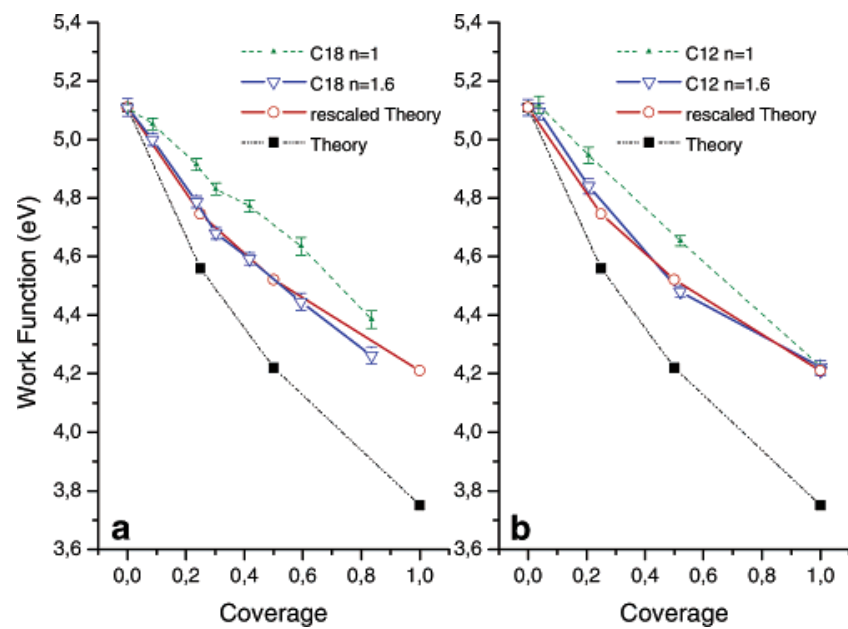

Figure 8. Work-function for $\mathrm{C} 18$ (a) and $\mathrm{C} 12$ (b) as obtained from corrected experimental values determined using eq (5) for $n=1$ and $n=1.6$. In both panels the theoretical results from DFT calculations for $\mathrm{CH}_{3} \mathrm{~S}$ at different methanthiolate coverages are also shown. The "rescaled theory" data are obtained by multiplying the work-function variation by the fixed factor 1.5 (see text for details).

this allows for the maximum amount of "contamination" to occur. The presence of SAM should reduce this effect since it "shields" the interface from the contaminating molecules. Hence we expect, for data reported in Figure 5, that at $t=0$ the effect of the contamination will be maximum while at $t>600 \mathrm{~s}$ this effect should be reduced to zero (or at least minimized) as we have complete coverage/formation of a SAM. Since there is no a priori reason why we should expect the growth kinetics of the SAM as monitored by the wetting technique to follow the same "work-function" kinetics, we write

$$
\text { offset }=\left(\Phi_{\mathrm{Au}}^{\mathrm{true}}-\Phi_{\mathrm{Au}}^{\mathrm{exp}}\right) e^{-k_{\Phi} t}=\left(\Phi_{\mathrm{Au}}^{\mathrm{true}}-\Phi_{\mathrm{Au}}^{\mathrm{exp}}\right)(1-\theta)^{n}
$$

where

$$
n=\frac{k_{\Phi}}{k_{\mathrm{L}}}
$$

where $\mathrm{k}_{\Phi}$ is the decay rate of the work-function offset. Hence the corrected value $\left(\Phi_{\text {SAM }}^{\text {true }}\right)$ for the work-function of gold coated with the SAM will be given by the relationship

$$
\Phi_{\mathrm{SAM}}^{\text {true }}=\Phi_{\mathrm{SAM}}^{\exp }+\text { offset }
$$

The corrected value $\Phi_{\text {SAM }}^{\text {true }}$ for the work-function of C12 and C18 are reported in Figure 8 together with the calculated values for $\mathrm{CH}_{3} \mathrm{~S}$-covered gold SAMs, as a function of $\theta$. We report the corrected values with $n=1$ and $n=1.6$ that is obtained by fitting to the theoretical data (see Theoretical Results). For both $\mathrm{C} 12$ and $\mathrm{C} 18$ the experimental work-function reach $4.2 \mathrm{eV}$ at maximum coverage, showing that the chain length has a marginal effect on the work-function. The theoretical results overestimate the workfunction variation between clean gold and the full covered substrate (in our case, by a factor 1.5), due to long-range interaction that are present in a full coverage, perfectly ordered SAM, such as the one modeled in the calculations. ${ }^{44}$ In Figure 8, we also report the "rescaled" theoretical data (obtained multiplying the work-function variation by the fixed factor 1.5 in order to reproduce the experimental value at full coverage) to avoid the theoretical overestimation of work-function variation maintaining its nonlinear behavior.
As it can be seen in Figure 8 the value of $n$ strongly affects the variation of the corrected value $\Phi_{\text {SAM }}^{\text {true }}$ with the coverage, but the agreement with the rescaled theoretical data is best when $n$ $=1.6$, implying that slightly different kinetics are observed when measuring the contact angle, $\theta(\mathrm{t})$, versus the case when the workfunction, $\Phi(\mathrm{t})$, is monitored.

This nonlinearity in eq 5 ( $n$ different from unity) may originate from different intermolecular interactions between the adsorbed molecules and the substrate as a function of coverage, and it can also be related to the polarizability. ${ }^{62,63}$ However, $n$ is a parameter in eq 5, and our experiments are performed on much longer molecules $(\mathrm{C} 18)$ than were considered in the theoretical calculations (C1). Whether this nonlinear dependence of the workfunction on the coverage persists in the calculations when longer chain lengths adsorbates are used requires further investigation. Currently, direct comparison between calculations and experiments is not possible for two reasons: experimentally our attempts to perform similar LEPIS measurements for the C6 were not successful because the disorder of the adsorbed organic layers produced by dipping is typically too large. ${ }^{15,55}$ In order to study the kinetics of the formation of SAMs for these small chains (C6 and $\mathrm{C} 1$ ), the preparation of the substrates must be performed under UHV conditions ${ }^{42}$ presently not possible in our setup. Moreover, theoretically the computational costs of performing plane-wave DFT calculations for large molecules at low coverages are unaffordable. In the calculations, the values of the workfunction for $\mathrm{CH}_{3} \mathrm{~S}$ and $\mathrm{C} 6$, for a complete coverage, are almost identical. This observation confirms the experimental findings, obtained both in this work and in previous studies, ${ }^{17}$ indicating that the induced change in the work-function is only slightly affected by the chain length and that the main variation is due to the interface properties and the SAM organization. The calculated values for the work-function drop by $\Delta \Phi=-1.36$ $\mathrm{eV}$ and $\Delta \Phi=-1.49 \mathrm{eV}$ for complete coverage. These changes can be considered as upper limits with respect to experimental results, being calculated for a complete monolayer perfectly ordered on the surface. ${ }^{44}$ However, values of $\Delta \Phi$ as large as 1.4 $\mathrm{eV}^{17}$ have been reported as an experimental upper limit for $\mathrm{C} 16$.

Another interesting result concerns the change in the workfunction in the case of the less stable adsorption configurations (Figure 6b,d). Here, one obtains an additional drop in the workfunction of $0.8 \mathrm{eV}$, with respect to the stable adsorption geometry. This large difference confirms the sensitivity of $\Phi$ ab initio calculations to the details of the interface. Indeed, for the C6, a variation of the molecular orientation with respect to the surface (from Figure 6a,b), within the same adsorption site, is enough to induce a change of $0.82 \mathrm{eV}$ in $\Phi$. Therefore, we cannot exclude the possibility that a calculation that includes slightly different orientations of the molecules in the same supercell, as in the $c(4 \times 2)$ case, would yield more refined values for the $\Phi$ changes, compared to those obtained here using the single-chain hexagonal model. However, this type of calculation requires a quite large supercell and a lot of different possible configurations.

It is important to realize that the large effect on the $\Phi$ variation, observed in the case of the less stable configurations, is consistent with the changes in $\Phi$ induced by adsorbates that interact weakly with the metal substrate. A recent theoretical investigation ${ }^{42}$ on work-function modifications induced by thiols on gold showed a variation in the order of $1 \mathrm{eV}$ for methylthiol and $1.5 \mathrm{eV}$ for dimethylthiol.

(62) Fukagawa, H.; Yamane, H.; Kera, S.; Okudaira, K. K.; Ueno, N.; Phys. Rev. B 2006, 73, 041302R.

(63) Bussolotti, F.; Betti, M. G.; Mariani, C. Phys. Rev. B 2006, 74, 125422. 


\section{Conclusions}

In this paper, we introduced the LEPIS as a method for monitoring adsorption kinetics. The method is relatively simple and provides work-function information. As a first application, the LEPIS method has been used to verify the adsorption kinetics of alkanethiols on gold. The results are consistent with data obtained before by other techniques. By monitoring the changes in the work-function as a function of contact angle, the selfassembly process can be followed. We found that the adsorption kinetics using CA measurements can be described using a Langmuir model, but the work-function kinetics show a slight deviation from this mechanism. Theoretical modeling of the experimental results has been presented and the agreements with the experimental observations have been addressed.
Acknowledgment. The experimental work was performed at the Ultraviolet Laser Facility operating at IESL-FORTH and was supported in part by the European Commission through the Research Infrastructures activity of FP6 ("Laserlab-Europe" RII3CT-2003-506350). The work was supported by the EU Growth Program (SENTIMATS G5RD-CT-2001-00569). We thank the graduate program Applied Molecular Spectroscopy (EPEAEK) and PENED (01ED419-2001). Theoretical calculations were performed at the ISUFI-CACT (Lecce). We thank the SPACI consortium for providing computational facilities. The theoretical work was supported by the European Project SA-NANO (Contract STRP 013698).

LA063471T 